\title{
Antitumor Enhancement by Irradiated Haploidentical Donor Lymphocyte Infusion of Mice with Melanoma
}

\author{
Yingjiao Zhu Xiaolan Laib $^{\mathrm{a}}$ Shen Zhaoc ${ }^{c}$ Jianwei Zheng ${ }^{\mathrm{d}, \mathrm{e}} \quad$ Xinli Wang ${ }^{\mathrm{d}}$ \\ Xiaojiao Weng ${ }^{d}$ Jieyu Lic Yubin Ye ${ }^{c, e, f}$ Qiang Chen ${ }^{d, e, f} \quad$ Xiaoyan Lin ${ }^{d, e}$
}

aDepartment of Medical Oncology, The Third Affiliated Hospital of Fujian Medical University, Fuzhou, Fujian, ${ }^{b}$ Department of Medical Oncology and Radiotherapy, Ningde Municipal Hospital Affiliated to Fujian Medical University, Ningde, Fujian, 'Department of Immuno-Oncology Laboratory, Fujian Provincial Cancer Hospital, Fuzhou, Fujian, 'Department of Medical Oncology, Fujian Medical University Union Hospital, Fuzhou, Fujian, eStem Cell Research Institute of Fujian Medical University and Fujian Key Laboratory of Translational Cancer Medicine, Fuzhou, Fujian, 'Fraduate School of Education, Fujian Medical University, Fuzhou, Fujian, China

\section{Key Words}

Immunotherapy • Donor lymphocytes infusion • Melanoma • Mouse

\begin{abstract}
Background: Previous researches have reported that donor lymphocyte infusion (DLI) provides a new approach for the treatment of hematological malignancies and some solid tumors. The present study was designed to discuss the antitumor effect on mice with melanoma and possible involvement of the mechanism of haploidentical DLI in CB6F1 mice $\rightarrow$ CC3HF1 mice (F1 $\rightarrow F 1)$ mouse model. Methods: An F1 $\rightarrow \mathrm{F} 1$ haploidentical infusion model was established. CB6F1 mice $(\mathrm{H}-2 \mathrm{~b} / \mathrm{d})$ bearing melanoma were used as recipients. CC3HF1 mice $(\mathrm{H}-2 \mathrm{~d} / \mathrm{k})$ were used as donors. Changes in tumor volume and mice survival, host-derived lymphocytes proliferation, cytotoxicity, donor cell survival in vivo, histopathological examination of important organs, and the secretion Th1/Th2 cytokines were analyzed. Results: Irradiated haploidentical DLI combined with low-dose cyclophosphamide (Cy) chemotherapy induced an antitumor effect on mice with melanoma using the F1 $\rightarrow$ F1 infusion model. Graft-versus-host disease (GvHD) was not obvious in any DLI-treated groups. Donor lymphocytes disappeared within 5 days after infusion, while the antitumor effect continued to be observed. Moreover, the DLI-treated groups showed a significant increase in the secretion of Th1 cytokines, including IFN- $\gamma$ and IL2 , and an enhanced proliferation of $\mathrm{CD} 8^{+} \mathrm{T}$ lymphocytes and NK cells. Conclusions: Irradiated haploidentical DLI without bone marrow transplantation offers a safe, feasible, and effective approach in the treatment of melanoma.
\end{abstract}

Y. Zhu and X. Lai contributed to this study equally. 


\section{Cellular Physiology Cell Physiol Biochem 2015;35:2181-2191 \begin{tabular}{ll|l} 
DOI: 10.1159/000374023 & $\begin{array}{l}\text { O 2015 S. Karger AG, Basel } \\
\text { www.karger.com/cpb }\end{array}$ \\
\hline
\end{tabular} \\ Zhu et al.: Antitumor Enhancement by Irradiated Haploidentical Donor Lymphocyte}

\section{Introduction}

As an important strategy of adoptive cell immunotherapy (ACI), donor lymphocyte infusion (DLI) after allogeneic hematopoietic stem cell transplantation (allo-HSCT) plays an increasingly important role in comprehensive cancer therapy. In early 1987, Slavin et al. introduced a treatment modality called DLI for patients who failed bone marrow transplantation [1]. Subsequently, DLI has been used to induce or reinforce graft-versustumor (GvT) response in the treatment of hematological malignancies and some solid tumors in mice and patients [2-5]. Nevertheless, high risk of lethal graft-versus-host disease (GvHD) is the major problem of DLI, which can reduce the effect of GvT [6]. Moreover, this treatment currently confronts obvious shortcomings such as high cost, limited donor sources, and low expansion ratio, which extensively hinder its clinical use. Previous studies have indicated that a preparative regimen (nonmyeloablative preparative lymphodepletion) as a valuable strategy to prevent GvHD in cancer patients undergoing treatment $[7,8]$.

Previous studies have shown that irradiation suppresses the proliferation of lymphocytes without affecting their antitumor cytotoxicity; therefore, it can preserve antitumor activity as well as limit the GvHD response [9-11]. In the past several years, several investigators have reported that MHC-haploidentical DLI could also generate an effective antitumor response compared with MHC-matched donor cells. MHC-haploidentical donors can be derived from the parents, children, siblings, and cousins of cancer patients. Shi et al. showed that inactivated haploidentical donor lymphocytes after adoptive transfer enhanced the GvT effect in a lung tumor model, inhibited tumor growth, and prolonged the survival of tumorbearing mice [12].

The incidence of melanoma, an immunogenicity tumor, has been increasing at unprecedented speed. DLI offers a new approach in the treatment of melanoma. However, definitive published data to guide its clinical practice is limited, and extensive researches on its applications are warranted. Adoptive cell immunotherapy offers a new approach for the treatment of melanoma, with evidence of durable antitumor effect in clinical trials. However, the tumor microenvironment is strongly associated with a complicated immunosuppressive network that often leads to immunological ignorance, which in turn limits the efficacy of various treatment schemes. Therefore, we established the subcutaneous tumor and pulmonary metastasis models to discuss the antimelanoma effect and possible involvement of the mechanism of irradiated haploidentical DLI. As MHC-haploidentical donors are more easily acquired than MHC-identical donors solving the problem of shortage of donor sources for clinical applications, we chose the $\mathrm{F} 1(\mathrm{H}-2 \mathrm{~d} / \mathrm{k}) \rightarrow \mathrm{F} 1(\mathrm{H}-2 \mathrm{~b} / \mathrm{d})$ mouse model, which is one of our innovations for the present study. We observed that the irradiated haploidentical DLI without allo-HSCT was highly effective in enhancing antimelanoma immunity and suppressing tumor growth in subcutaneous melanoma and pulmonary metastasis models.

\section{Materials and Methods}

\section{Tumor cell lines and mice}

The B16-F10 cell line (H-2b) was purchased from the Institute of Cytobiology, Chinese Academy of Sciences (Shanghai, China). This cell line was cultured in DMEM medium supplemented with $10 \%$ heatinactivated fetal bovine serum (FBS), $100 \mu \mathrm{g} / \mathrm{mL}$ streptomycin, and $100 \mathrm{IU} / \mathrm{mL}$ penicillin at $37^{\circ} \mathrm{C}$ with $5 \%$ $\mathrm{CO}_{2}$ in a humidified atmosphere and harvested using $0.25 \%$ trypsin-EDTA. Female CC3HF1 $(\mathrm{C} 3 \mathrm{H} \times \mathrm{Balb} / \mathrm{c})$ and female CB6F1 (C57BL/6×Balb/c) mice within the age range of 6-8 weeks were obtained from the Vital River Laboratory Animal Technology Co., Ltd. (Beijing, China) and kept in specific pathogen-free conditions. An F1-F1 haploidentical infusion model was established. CC3HF1 mice (H-2d/k) were utilized as donors, whereas CB6F1 mice (H-2b/d) were used as recipients. The Animal Ethics Committee of Fujian Medical University approved all the animal experiments conducted in the present study.

\section{KARGER}




\section{Cellular Physiology Cell Physiol Biochem 2015;35:2181-2191 \begin{tabular}{l|l|l|} 
and Biochemistry & $\begin{array}{l}\text { Dol: 10.1159/000374023 } \\
\text { Published online: April 07, } 2015\end{array}$ & $\begin{array}{l}\text { 2015 S. Karger AG, Basel } \\
\text { www.karger.com/cpb }\end{array}$ \\
\cline { 2 - 3 }
\end{tabular}

Tumor models and donor lymphocyte infusion

Spleens were removed aseptically from donor CC3HF1 mice and then minced with scissors. A total of $8.0 \times 10^{7}$ splenocytes were isolated from the minced spleens by passing through a cell strainer, followed by lysis of the red blood cells. A total of $3.0 \times 10^{7} \mathrm{~T}$ lymphocytes were MACS-isolated from donor splenocytes per mouse by using Pot T cell Isolation Kit II mouse (Miltenyi Biotec, Germany). Then, all the cells were irradiated with 5 Gy and adjusted to a density of $1 \times 10^{8}$ viable cells $/ \mathrm{mL}$ in phosphate-buffered saline (PBS).

CB6F1 mice were inoculated subcutaneously in the left hindlimb and injected intravenously by the tail vein with $1 \times 10^{5}$ B16-F10 melanoma cells on Day 0. Cyclophosphamide (Cy) dissolved in PBS (20 mg/ $\mathrm{mL}$ ) was injected intraperitoneally at a dose of $100 \mathrm{mg} / \mathrm{kg}$ approximately 3 days later (Day 3). On Day 6, the mice received DLI treatment. Mice were monitored every 3 days, including body weight measurements and survival time. The sizes of the subcutaneous tumors were determined by measuring with calipers. Subcutaneous resected tumor weights were measured on Day 22. For the pulmonary metastasis model, all the mice were sacrificed on Day 18 and the resected lungs were weighed. Subcutaneous tumor volume was calculated using the formula: tumor volume $\left(\mathrm{mm}^{3}\right)=$ width ${ }^{2} \times$ length $/ 2\left(\mathrm{~mm}^{3}\right)$.

Assessment of GvHD

GvHD was monitored by screening for GVHD-related symptoms and confirmed by histology of the skin, lungs, small intestine, and liver. On day 13 after DLI treatment, 3 mice from each group were sacrificed, and their skin, lungs, small intestines, and livers were subjected to histopathological examination. The GvHD-related symptoms were monitored daily and assessed according to established criteria that summed up changes in five clinical parameters (maximum score of 10): weight loss, posture (hunching), activity, fur texture, and skin integrity [13].

\section{Measurement of cytokine levels}

Serum samples were collected from each group of treated mice at 3 and 10 days after DLI treatment. The detection of IFN- $\gamma$, IL-2, IL-4, and IL-10 were measured using specific enzyme-linked immunosorbent assay (ELISA) kits for mouse IL-2, IFN- $\gamma$, IL-4, and IL-10 (R\&D, USA), according to the manufacturer's protocol.

Flow cytometric analysis of donor cells and recipient splenocyte subsets

Flow cytometry was used to analyze the survival time of donor lymphocytes as well as changes in recipient splenocyte subsets at indicated time points. The spleens of the treated mice were harvested at -1 , $1,3,4,5,7,13$, and 18 days after DLI treatment. After single-cell suspensions of splenocytes were prepared, the number of splenocytes was counted, and the erythrocytes were lysed. Then, the cell suspensions were stained with fluorescein isothiocyanate (FITC)-conjugated anti-mouse H-2Kk, CD4, CD8, CD49b/Pan-NK, and CD19 mAb or phycoerythrin (PE)-conjugated anti-mouse H-2Kb, and CD3 mAb (BD, USA) at room temperature for $20 \mathrm{~min}$. After staining, the cells were washed with PBS and analyzed on a flow cytometer (Beckman, USA) according to the manufacturer's instruction. The number of each lymphocyte subsets in the host spleens at indicated time points was calculated: Number of donor-derived splenocytes $=[$ (spleen cell count $\left.) \times\left(\% \mathrm{H}-2 \mathrm{Kk}^{+}\right)\right]$; and number of recipient-derived splenocytes $=[$(spleen cell count $) \times\left(\% \mathrm{H}-2 \mathrm{~Kb}^{+}\right) \times$ (\% $\mathrm{CD}^{+}, \mathrm{CD}^{+}, \mathrm{CD} 49 \mathrm{~b} / \mathrm{Pan}-\mathrm{NK}^{+}$or CD19+].

\section{Cytotoxicity assays}

As early as on day 3 and day 10 after DLI treatment, the tumor-bearing CB6F1 mice were sacrificed and spleens were freshly harvested. The cytotoxicity of splenocytes was analyzed using a Cytotoxicity Detection Kit (Roche, Switzerland), according to the manufacturer's instructions. Splenocytes from recipient mice were used as effector cells and B16-F10 cells were utilized as target cells. Effector cells were added to target cells at a ratio of 15:1, 30:1, and 60:1, respectively (tested in triplicate).

\section{Statistical analysis}

All data are presented as means \pm SD/SE. One-way ANOVA was used to compare continuous variables among inter-groups, and the Student-Newman-Keuls test was used to compare differences between 2 groups. The statistical significance of the survival data was determined by using the log-rank test. A value of $\mathrm{p}<0.05$ was considered significant. Statistical analysis was conducted by using the software, SPSS 22.0. 

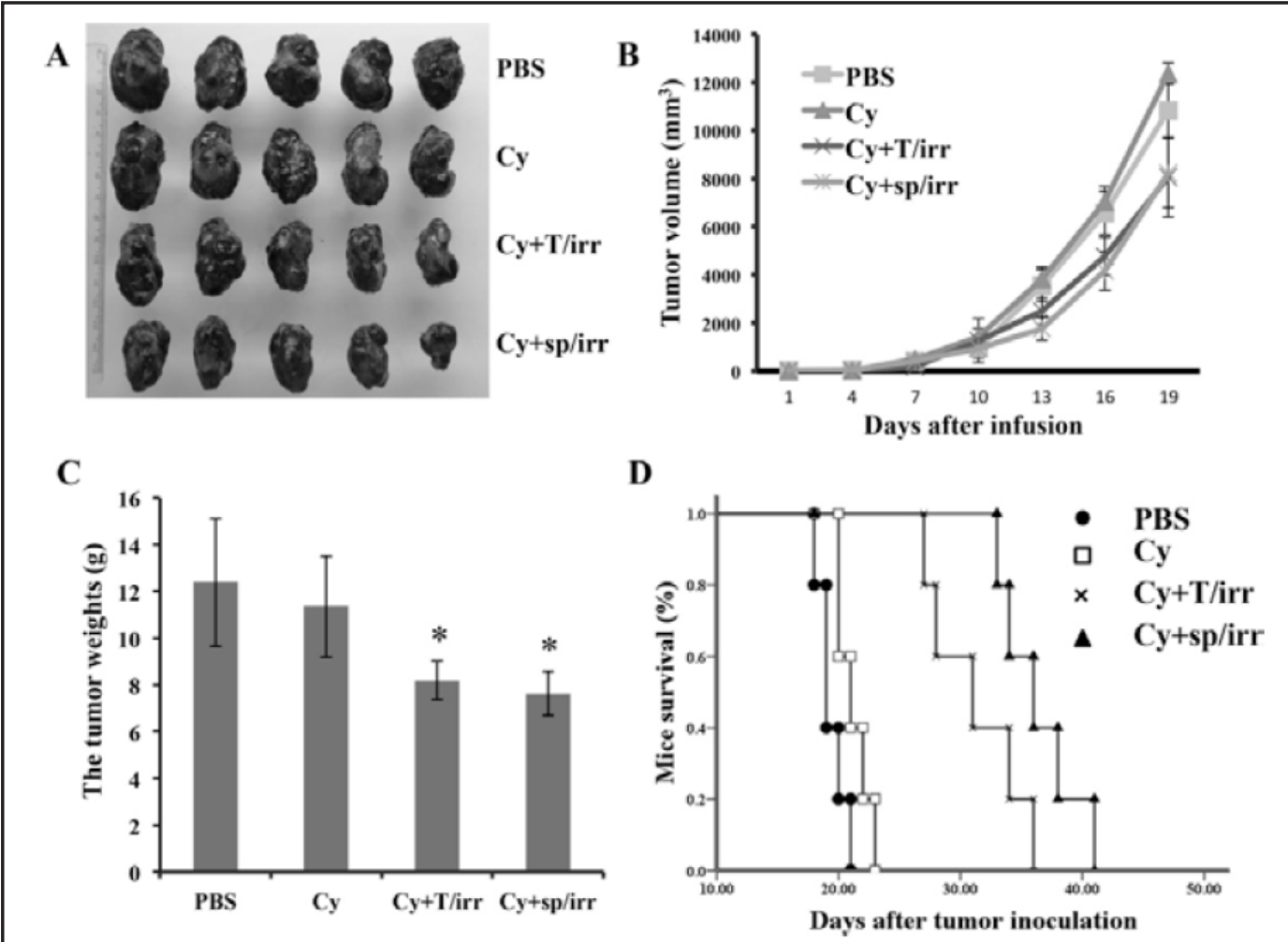

Fig. 1. The antitumor effects of DLI in a mouse subcutaneous tumor model. All the mice bearing melanoma were divided into four groups (PBS, mice receiving intravenous PBS; Cy, mice that received intravenous PBS after a intraperitoneal of cyclophosphamide; Cy+T/irr, cyclophosphamide combined with $3.0 \times 10^{7}$ irradiated T cells separated form CC3HF1 splenocytes; and Cy +Sp/irr, cyclophosphamide combined with $8.0 \times 10^{7}$ irradiated CC3HF1 splenocytes). (A) The subcutaneous tumor. (B) The growth curves of inoculated subcutaneous tumor of mice bearing melanoma. The tumor growth was obviously suppressed in mice treated with $\mathrm{Cy}+\mathrm{T} / \mathrm{irr}, \mathrm{Cy}+\mathrm{sp} / \operatorname{irr}(\mathrm{P}<0.05)$. (C) The tumor weights at 16 days after DLI treatment. $* \mathrm{P}<0.05$ compared to the PBS group. (D) The MST of mice after DLI treatment. The data are presented as mean \pm SD; $\mathrm{n}=5$ mice in all groups.

\section{Results}

Irradiated haploidentical DLI suppresses tumor growth and lung metastasis

We first used the B16-F10 subcutaneous tumor model to assess the antitumor effects of irradiated haploidentical DLI (Fig. 1A). Donor cells infusion administration systemically suppressed tumor growth, as indicated by the assessment of tumor volume and weight. As shown in Fig. 1B-D, no significant differences in tumor growth, weight, or mean survival time (MST) between PBS and Cy groups were observed ( $p>0.05)$. On the other hand, tumor growth and weight were significantly suppressed, and MST was obviously prolonged in the $\mathrm{Cy}+\mathrm{T} /$ irr and $\mathrm{Cy}+\mathrm{sp} /$ irr groups compared to the PBS or Cy groups. Moreover, tumor growth, weight, or MST were the same in the $\mathrm{Cy}+\mathrm{T} /$ irr and $\mathrm{Cy}+\mathrm{sp} /$ irr groups $(\mathrm{P}>0.05)$.

An artificial lung metastasis model was also performed to examine the antimetastatic effect of irradiated haploidentical DLI (HLI). As shown in Fig. 2A-B, the average lung weights of Cy group were significantly greater than those of the DLI-treated groups. Mice treated with sp/irr and Cy+sp/irr showed a longer MST than the Cy group (Fig. 2C). However, the sp/ irr group showed greater lung weights and longer MSTs than the Cy+sp/irr groups $(\mathrm{P}<0.05)$. 


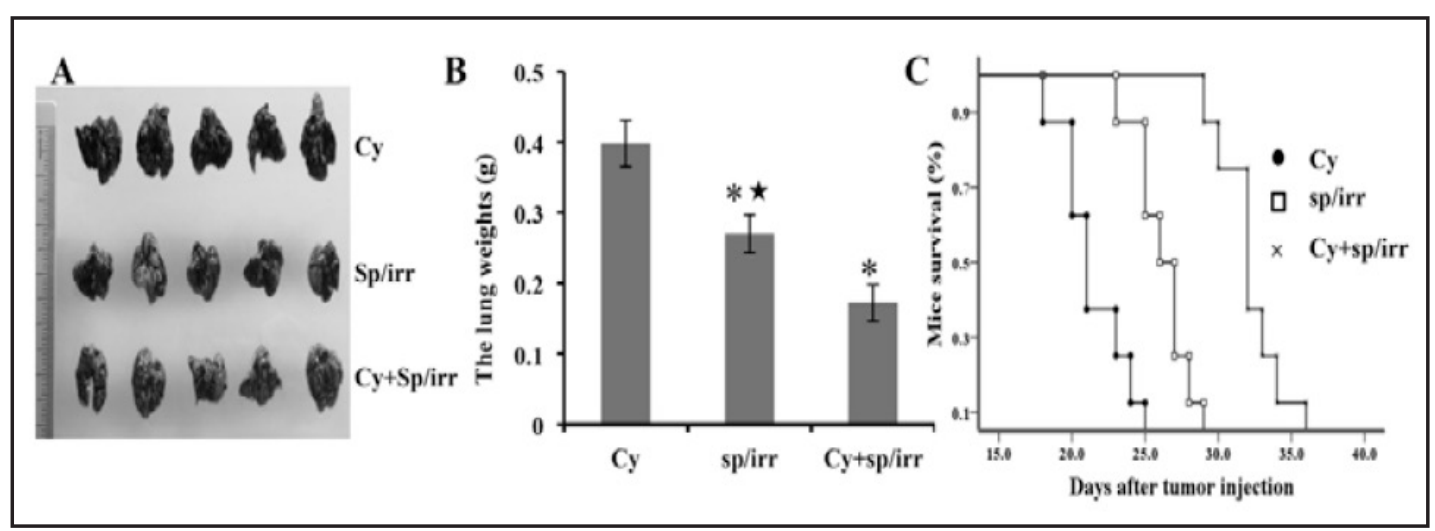

Fig. 2. The antitumor effects of DLI in a pulmonary metastasis tumor model. All the melanoma-bearing mice were divided into three groups: $\mathrm{Cy}$ (mice received a intraperitoneal of cyclophosphamide), Sp/irr $\left(8.0 \times 10^{7}\right.$ irradiated CC3HF1 splenocytes), aFig.nd Cy+Sp/irr (cyclophosphamide combined with $8.0 \times 107$ irradiated CC3HF1 splenocytes). (A) The pulmonary metastasis tumor. (B) Lung weight measurements. *P $<0.05$ compared to the Cy group; ${ }^{\star} \mathrm{P}<0.05$ compared to the $\mathrm{Cy}+\mathrm{Sp} /$ irr group. (C) MST of mice after DLI treatment.

Fig. 3. GvHD pathological manifestations in the liver, lungs, intestine, and skin on day 13 after DLI treatment of a subcutaneous tumor model (HE, original magnification $\times 200$ ).

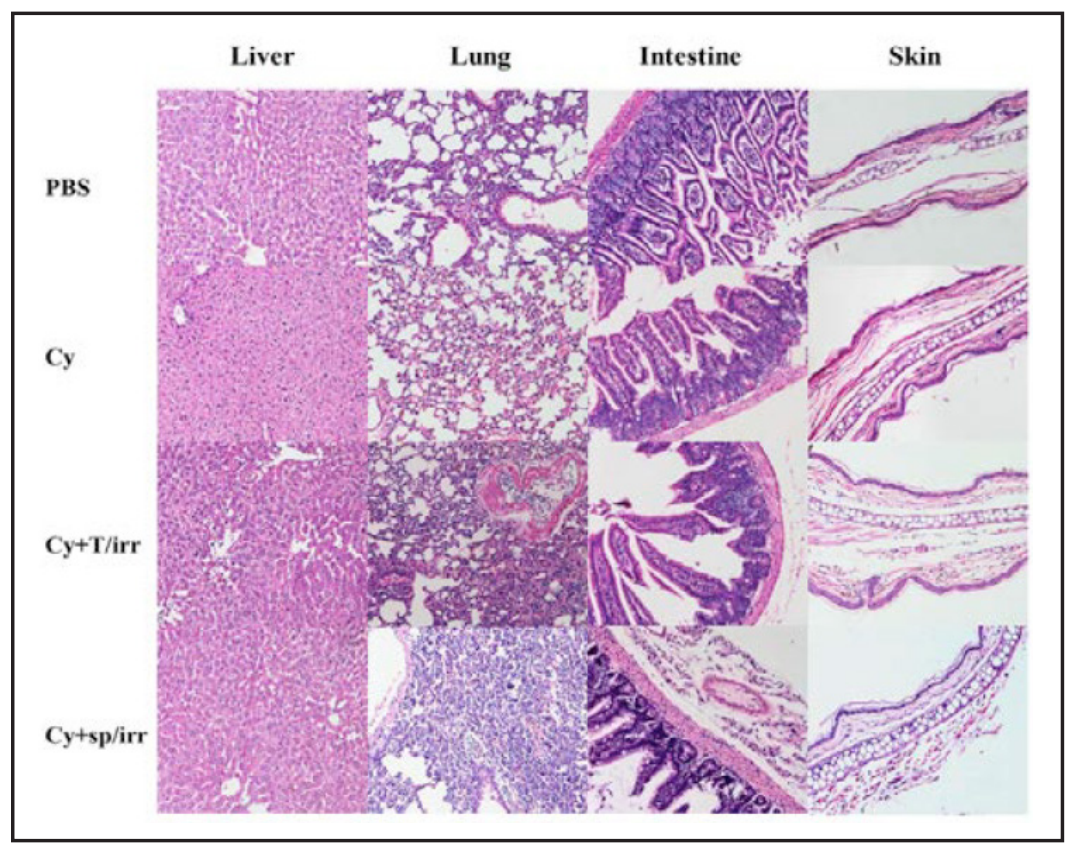

Table 1. Comparison of GvHD values after DLI treatment

\begin{tabular}{clllll}
\hline \multirow{2}{*}{ Time (days) } & \multicolumn{4}{c}{ GvHD score values } & \multirow{2}{*}{ P value } \\
& PBS & Cy+T/irr & Cy+sp/irr & \\
\hline 3 & $0.20 \pm 0.45$ & $0.40 \pm 0.55$ & $0.6 \pm 0.55$ & 0.493 \\
8 & $1.60 \pm 0.55$ & $1.80 \pm 0.84$ & $2.0 \pm 0.71$ & 0.679 \\
13 & $3.60 \pm 0.55$ & $4.00 \pm 0.71$ & $3.8 \pm 0.45$ & 0.564 \\
\hline
\end{tabular}

\section{Assessments of GvHD after DLI treatment in B16-F10-melanoma-bearing mice}

The infusion of donor allogeneic lymphocytes can induce GvHD-like reactions in vivo. Therefore, we assessed the degree of GvHD by using an established scoring system in a subcutaneous tumor model. As shown in Table 1, the GvHD-related clinical symptoms were not apparently different among the groups after DLI treatment. On the other hand, no overt lesions in the skin, liver, lungs, or intestines were observed compared to PBS-treated mice on histopathological examination (Fig. 3).

\section{KARGER}


Fig. 4. Survival of donor-derived lymphocytes in recipient $\mathrm{CB} 6 \mathrm{~F} 1$ mice $(\mathrm{n}=5$ for all groups).
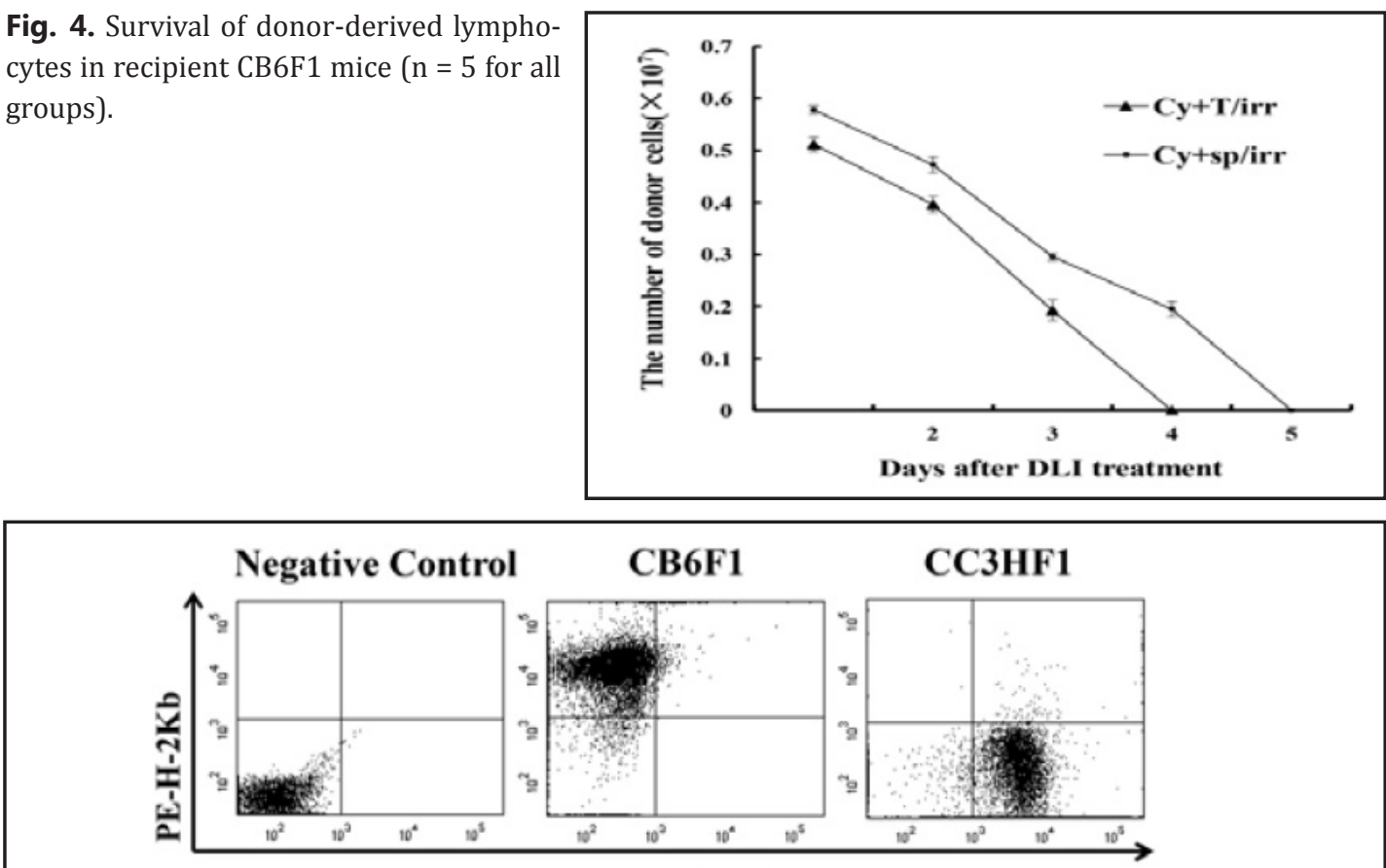

FITC-H-2Kk

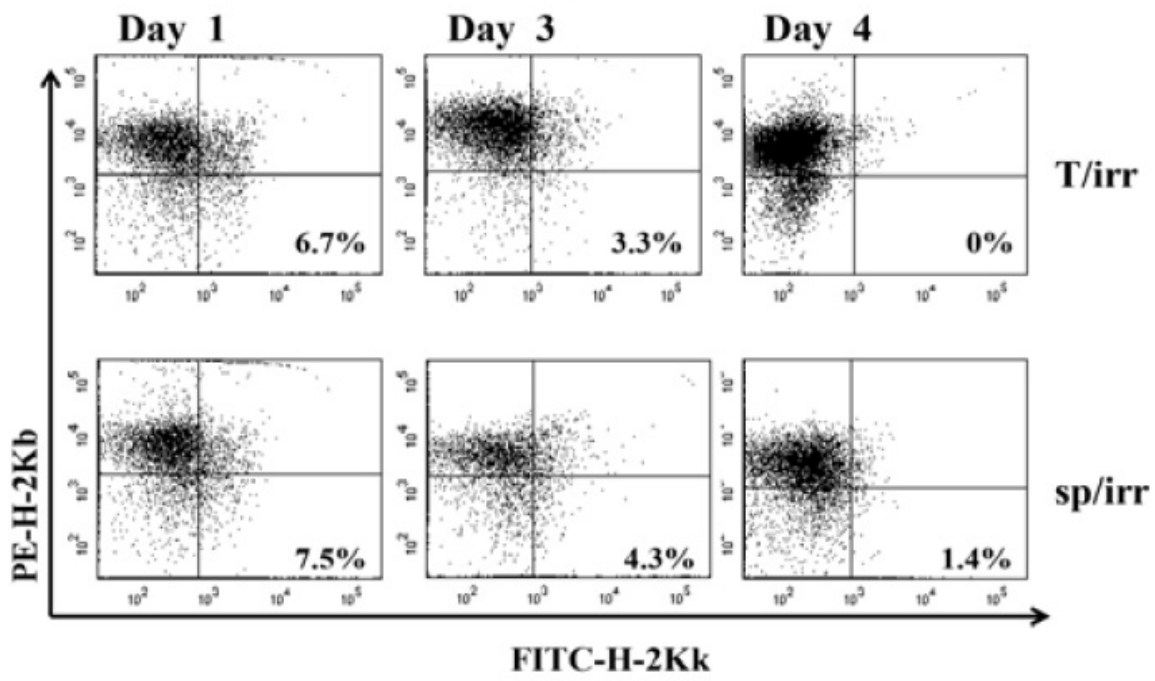

Fig. 5. Donor-derived lymphocytes persist in recipients. H-2Kb isotype expression of splenocytes derived from recipient CB6F1 mice. H-2Kk isotype expression of splenocytes derived from donor CC3HF1 mice. $\mathrm{H}-2 \mathrm{~Kb}$ or $\mathrm{H}-2 \mathrm{Kk}$ expression on splenocytes is determined by FACS ( $\mathrm{n}=3$ for all groups). The experiment was conducted in triplicate.

Transient persistence of irradiated MHC-haploidentical donor splenocytes in recipients after transfer

To verify the duration of donor splenocytes in vivo, splenocyte (CB6F1 recipient) were harvested on days 1, 2, 3, 4, and 5 after DLI treatment. All the donor-derived splenocytes from the DLI-treated groups disappeared within 5 days (Fig. 4). As shown in Fig. 5B, at day 1 after injection, the percentage of $\mathrm{H}-2 \mathrm{Kk}$-positive lymphocytes from $\mathrm{Cy}+\mathrm{sp} / \mathrm{irr}-$ and $\mathrm{Cy}+\mathrm{T} /$ irr-treated recipients was $7.5 \%$ and $6.7 \%$, respectively. However, the percentage of $\mathrm{H}-2 \mathrm{Kk}$ positive cells dramatically decreased after 4 days. Although the MST of donor-derived lymphocytes in the Cy+sp/irr group was longer than that in the Cy+T/irr group, this was not statistically significant $(\mathrm{P}>0.05)$. 


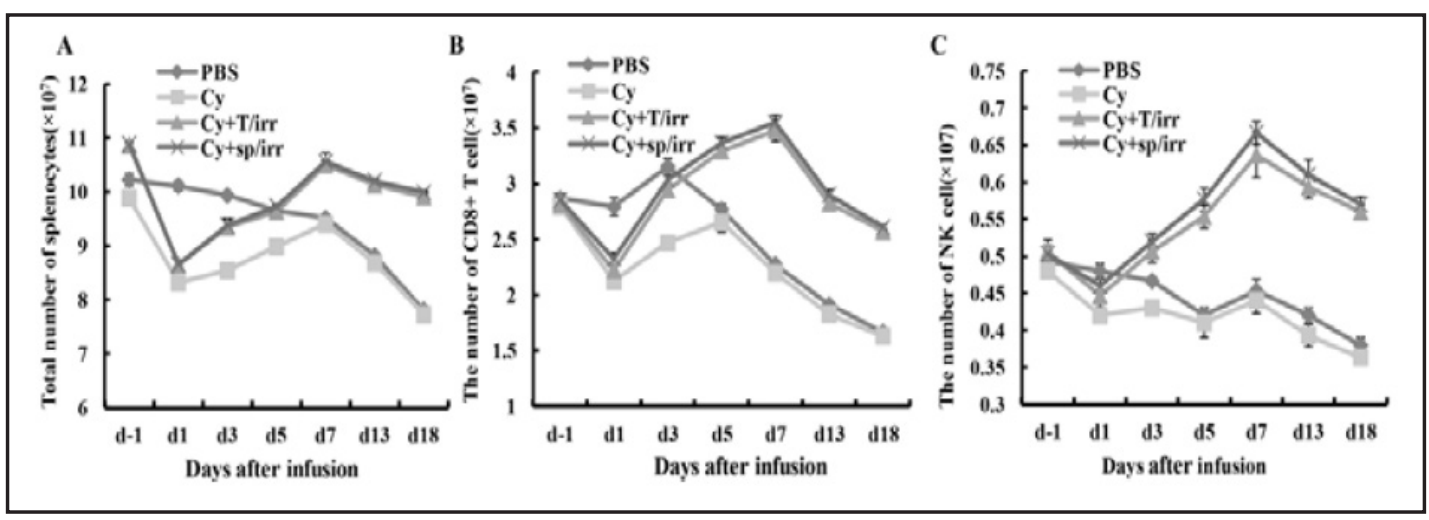

Fig. 6. Proliferation of the recipient-derived lymphocyte subset in vivo. (A) Total number of host-derived splenocytes. (B) Total number of host-derived CD8+ T cells. (C) Total number of host-derived NK cells. ( $n=3$ for each group). The experiment was conducted in triplicate.

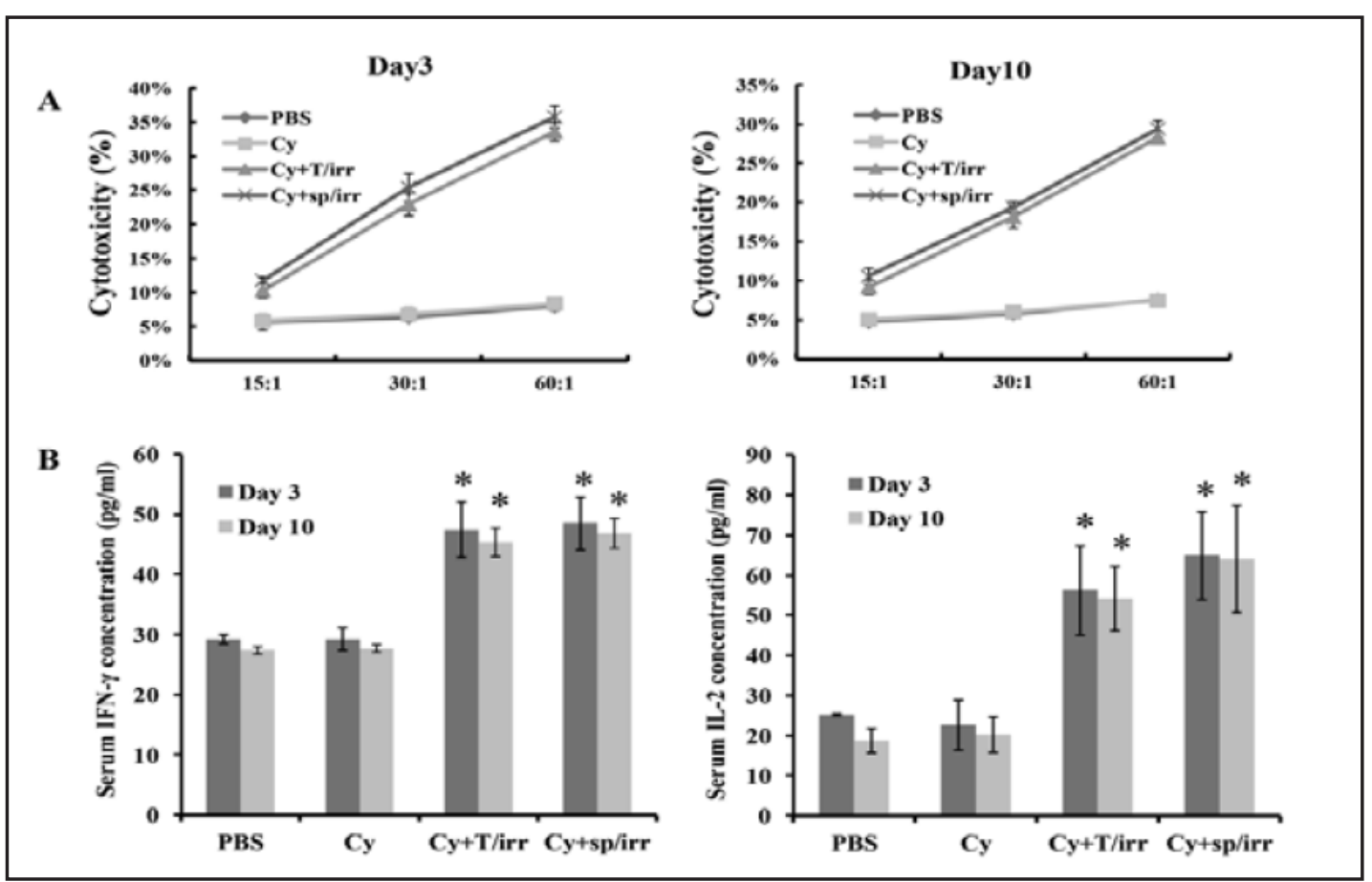

Fig. 7. Effect of DLI on cytotoxic assay and the serum levels of type 1 cytokines. (A) On Days 3 and 10 after tumor subcutaneous inoculation, the splenocytes were prepared and used as effector cells against B16-F10 cells at the indicated E:T ratio. (B) On Days 3 and 10 after tumor subcutaneous inoculation, serum was collected. The secretion of IL- 2 and IFN $-\gamma$ was determined by ELISA. ${ }^{*} \mathrm{P}<0.05$ compared to the PBS group ( $n=5$ for each group).

The proliferation of host lymphocytes contributes to the antitumor response

Next, we analyzed the change in host lymphocyte subsets by flow cytometry to further investigate the preliminary antitumor mechanism of irradiated MHC-haploidentical DLI. Our data showed that the total number of CB6F1 spleen cells and the number of host-type $\mathrm{CD} 4^{+} \mathrm{T}$ cells, $\mathrm{CD} 8^{+} \mathrm{T}$ cells, NK, and B cells of PBS and Cy groups reduced stably with tumor growth (Fig. 6). Interestingly, compared to the PBS or Cy groups, the total number of spleen cells and recipient $\mathrm{CD} 4^{+} \mathrm{T}$ and $\mathrm{B}$ cells slightly decreased (date not shown), whereas the total number of recipient CD8+ T cells and NK cells significantly elevated in the splenocytes from mice receiving DLI treatment (Fig.s 6B-C). No significant differences between $\mathrm{Cy}+\mathrm{T} /$ irr and $\mathrm{Cy}+\mathrm{sp} /$ irr groups were observed $(\mathrm{P}>0.05)$. 


\section{Cellular Physiology Cell Physiol Biochem 2015;35:2181-2191

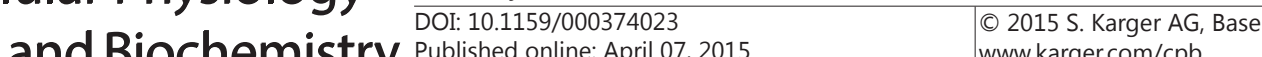 and $\frac{\text { Published online: April 07, } 2015}{\text { Zhu et al: Antitumor Enhancement by Irradiated Haploidentical Donor Lymphocyte }}$}

DLI-induced antitumor effect correlates with IL-2 and IFN- $\gamma$ secretion and cytolytic activity

We also detected cytolytic activity as early as on day 3 and day 10 after DLI treatment. Fig. 7A showed that DLI treatment enhanced the cytotoxicity of splenocytes compared to PBS-treated and Cy-treated mice. On the other hand, the cytotoxicity of splenocytes between $\mathrm{Cy}+\mathrm{T} /$ irr-treated and $\mathrm{Cy}+\mathrm{Sp} /$ irr-treated mice was not significantly different (Fig. 7A, P > 0.05). On day 10 after DLI treatment, the same level of cytolytic activity was observed even after the donor lymphocytes had disappeared (Fig. 7B). Then, as shown in Fig. 7B, DLI treatment remarkably enhanced the secretion of sera IL- 2 and IFN- $\gamma$ compared to the PBS and Cy groups, whereas DLI treatment had little effect on IL-4 and IL-10 production (data not shown). No significant differences in cytokine concentrations were observed between the $\mathrm{Cy}+\mathrm{T}$ /irr group and Cy+sp/irr group (Fig. 6B, P > 0.05). On day 10 after DLI treatment, the therapeutic effect could still be observed even after the donor lymphocytes had already disappeared.

\section{Discussion}

Data emerging from both mouse models and human clinical trials have indicated that a nonmyeloablative, lymphodepleting chemotherapy regimen improved the efficacy of donor lymphocytes after adoptive transfer $[8,14,15]$. Slavin's observation has provided evidence that a preparative regimen (antithymocyte globulin or Cy) followed by DLI is a valuable strategy for enhancing GvT, as well as in suppressing GvHD in heavily pretreated patients with advanced malignancies [16]. A single dose of cyclophosphamide was used as a preparative lymphodepletion $[10,17]$. To minimize GVHD effects, the concept of using irradiated donor lymphocytes to break tolerance and stimulate an anti-tumor response without the risk of GVHD has been attempted [18]. Previous studies have shown that irradiation preserves anti-tumor activity while limiting the GvHD response [9-11]. Donor irradiated lymphocytes cells also alter a patient's immunity, often resulting in a change in immunotolerance to the tumor and developing a delayed patient anti-tumor immune response [18]. However, the mechanism of irradiated donor lymphocytes to induce an antitumor response is still unclear. Thus, we chose a preparative regimen of low-dose Cy chemotherapy and 5 Gy radiotherapy. Our data showed that the GvT effect in the Cy+sp/irr group was significantly stronger than that observed in the sp/irr group in the pulmonary metastatic model. This result indicated that pretreatment with Cy could enhance the antitumor effect of DLI treatment. Moreover, we did not discover an overt GvHD response after DLI.

In the present study, we found that infusion of inactivated donor lymphocytes dramatically suppressed tumor growth as well as prolonged mice survival both in the subcutaneous tumor model and in the lung metastasis model. Collectively, these data indicate that donor lymphocytes could induce a better antitumor effect. T cells play an important role in the mechanism of antitumor immune responses. As expected, we found that the antitumor effect of spleen cells in the Cy+sp/irr group was stronger than that observed in the T cells in the $\mathrm{Cy}+\mathrm{T} /$ irr group in the subcutaneous tumor model, although this trend did not reach statistical significance. The results of the present study suggest that $T$ cells play a lead role in the DLI-induced antitumor effects that are in line with most studies [19]. Because direct infusion of spleen cells without separation was lower-cost, this strategy could perhaps be more beneficial for clinical applications.

Kamiryo et al. showed the involvement of tumor-reactive host CD8+ T cells, NK cells, and host-reactive donor-derived CD4+ T cells in the antitumor effects of nonmyeloablative hematopoietic stem cell transplantation in a murine bladder tumor model [20]. Likewise, our data revealed that host antitumor activity of DLI was reinforced after adoptive transfer of donor cells, resulting in the proliferation of host lymphocytes especially the CD8+ T and NK cells. Furthermore, it has been reported that donor cell infusion can induce the activation and proliferation of lymphocytes that is coupled with an upregulation of Th1 cytokines, thus 


\section{Cellular Physiology Cell Physiol Biochem 2015;35:2181-2191

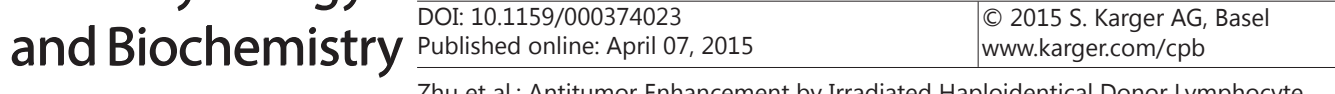

mediating innate and adaptive immune responses that participate in the antitumor activity of inactivated lymphocytes infusion [21]. Similarly, we found that DLI treatment remarkably enhanced the secretion of sera IL-2 and IFN- $\gamma$, whereas imparted little effect on the IL-4 and IL-10 production. Therefore, the "Th1 cytokine storm" is indispensable in activating the immune system in a tumor-bearing host. Meanwhile, we found that allogeneic donor lymphocytes were completely rejected by the host immune system within 5 days. However, on day 10 after DLI treatment, the concentrations of IL-2 and IFN- $\gamma$ remained high, in addition, the cytolytic activity of effector splenocytes from DLI-treated mice were sustained. Transient engraftment was sufficient to induce significant tumor regression. Therefore, we could deduce that the antitumor effect was principally dependent on the HvT response rather than the donor lymphocytes. However, T-cell expansion in vivo may also lead to the release of toxic levels of cytokines, including IL- 6 and INF- $\gamma$, often referred to as cytokine storms or cytokine release syndrome (CRS). Morgan et al. has previously shown that the serum sample of a metastatic colon cancer patient after chimeric antigen receptor infusion showed marked increases in INF- $\gamma$, IL-6, and IL-10, which was consistent with a cytokine storm, although the patient died 5 days after treatment [22]. Uncontrolled studies have demonstrated that immunosuppression using an anti-IL-6 receptor antibody, with or without corticosteroids, can reverse CRS [23]. However, it remains unclear whether cytokine storms is essential for maximal antitumor effect [23]. Mackall et al. have suggested that some cytokine storm toxicities are required to occur within the body, and further studies on the biology of these events are therefore warranted [23].

Taken together, the underlying mechanisms of DLI could be attributed to three reasons. First, allogeneic donor cells can be activated to mediate an allospecific effect of an anti-tumor response. Second, irradiated haploidentical DLI treatment could induce an alloresponse that breaks the host immune tolerance. Activation of the host immune system often results in the activation and proliferation of $\mathrm{CD}^{+} \mathrm{T}$ cells and NK cells. Ultimately, DLI induced both innate and adaptive immune responses against melanoma. It has been previously shown that antitumor activity is dependent on CD8 ${ }^{+} \mathrm{T}$ cells and NK cells; NK cells can induce proliferation and differentiation of $\mathrm{CD}^{+} \mathrm{T}$ cells into cytolytic effector T cells (CTL) and CD8 ${ }^{+}$ T cells can become activated via IL-12, which is produced by dendritic cells in response to IFN- $\gamma$ producing NK cells [24]. Third, previous studies have shown that the DLI-induced antitumor effect correlates with IL-2 and IFN- $\gamma$ secretion, which is in concordance with our study [25]. Alloreactivity is critical for antitumor responses. The host CD4+ Thl cells against donor MHC molecule are largely activated via direct and indirect recognition approaches during alloreactivity, giving rise to the dramatic increase of type 1 cytokines, including IL-2 and IFN- $\gamma[26,27]$. Finally, DLI facilitates enhancement of GvT via perforin-mediated cytotoxicity, fas-ligand cytolytic pathway, and possibly TNF-related apoptosis-inducing ligand with selective activity for malignant targets [28].

In summary, we showed that DLI is a safe, feasible, and effective approach for the treatment of melanoma in mouse models, without generating any detectable side effects. This treatment utilized a healthy donor immune system to activate the host immune system and prevent tumor progress, thus offering a new approach in the cure of patients with melanoma. However, its efficacy in activating grafted cells and reducing the risk of autoimmune response, controlling the number of infusion cells, establishing a good standard for the evaluation of a curative effect, and its effectiveness on other tumors still need to be further clarified.

\section{Disclosure Statement}

None.

\section{Acknowledgments}

This work was supported by Grant 2012J01351 for Natural Science Foundation from Fujian Province of China. 


\section{Cellular Physiology Cell Physiol Biochem 2015;35:2181-2191 \begin{tabular}{ll|l} 
and Biochemistry & $\begin{array}{l}\text { DOI: 10.1159/000374023 } \\
\text { Published online: April 07, 2015 }\end{array}$ & $\begin{array}{l}\text { O) 2015 S. Karger AG, Basel } \\
\text { www.karger.com/cpb }\end{array}$ \\
\cline { 2 - 3 }
\end{tabular} \\ Zhu et al.: Antitumor Enhancement by Irradiated Haploidentical Donor Lymphocyte}

\section{References}

1 Slavin S, Or R, Naparstek E, Ackerstein A, Weiss L: Cellular- mediated immunotherapy of leukemia in conjunction with au- tologous and allogeneic bone marrow transplantation in experimental animals and man. Blood 1988;72:407a.

2 Slavin S, Naparstek E, Nagler A, Ackerstein A, Kapelushnik J, Or R: Allogeneic cell therapy for relapsed leukemia after bone marrow transplantation with donor peripheral blood lymphocytes. Exp Hematol 1995;23:1553-1562.

3 Morecki S, Lindhofer H, Yacovlev E, Gelfand Y, Ruf P, Slavin S: Induction of long-lasting antitumor immunity by concomitant cell therapy with allogeneic lymphocytes and trifunctional bispecific antibody. Exp Hematol 2008;36:997-1003.

4 Geller MA, Cooley S, Judson PL, Ghebre R, Carson LF, Argenta PA, Jonson AL, Panoskaltsis-Mortari A, Curtsinger J, McKenna D, Dusenbery K, Bliss R, Downs LS, Miller JS: A phase II study of allogeneic natural killer cell therapy to treat patients with recurrent ovarian and breast cancer. Cytotherapy 2011;13:98-107.

5 Warlick ED, DeFor T, Blazar BR, Burns L, Verneris MR, Ustun C, Weisdorf DJ, Miller JS: Successful remission rates and survival after lymphodepleting chemotherapy and donor lymphocyte infusion for relapsed hematologic malignancies postallogeneic hematopoietic cell transplantation. Biol Blood Marrow Transplant 2012;18:480-486.

6 Stevanovic S, van Bergen CA, van Luxemburg-Heijs SA, van der Zouwen B, Jordanova ES, Kruisselbrink $\mathrm{AB}$, van de Meent M, Harskamp JC, Claas FH, Marijt EW, Zwaginga JJ, Halkes CJ, Jedema I, Griffioen M, Falkenburg JH: HLA class II upregulation during viral infection leads to HLA-DP-directed graft-versus-host disease after CD4+ donor lymphocyte infusion. Blood 2013;122:1963-1973.

7 Restifo NP, Dudley ME, Rosenberg SA: Adoptive immunotherapy for cancer: harnessing the T cell response. Nat Rev Immunol 2012;12:269-281.

8 Park YS, Bae JH, Son CH, Lee KS, Kim W, Jung MH, Yang K, Kim SH, Kang CD: Cyclophosphamide potentiates the antitumor effect of immunization with injection of immature dendritic cells into irradiated tumor. Immunol Invest 2011;40:383-399.

9 Sung AD, Chao NJ: Concise review: acute graft-versus-host disease: immunobiology, prevention, and treatment. Stem Cells Transl Med 2013;2:25-32.

10 Zhao S, Li JY, Zheng XB, Peng F, Ye YB, Chen Q: Irradiated haploidentical donor leukocyte infusions as an adoptive immunotherapy strategy to induce host-versus-tumor effects. Tumori 2011;97:522-531.

11 Sawitzki B, Brunstein C, Meisel C, Schumann J, Vogt K, Appelt C, Curtsinger JM, Verneris MR, Miller JS, Wagner JE, Blazar BR: Prevention of graft-versus-host disease by adoptive T regulatory therapy is associated with active repression of peripheral blood Toll-like receptor 5 mRNA expression. Biol Blood Marrow Transplant 2014;20:173-182.

12 Shi G, Zhou C, Wang D, Ma W, Liu B, Zhang S: Antitumor enhancement by adoptive transfer of tumor antigen primed, inactivated MHC-haploidentical lymphocytes. Cancer Lett 2014;343:42-50.

13 Cooke KR, Kobzik L, Martin TR, Brewer J, Delmonte J Jr, Crawford JM, Ferrara JL: An experimental model of idiopathic pneumonia syndrome after bone marrow transplantation: I. The roles of minor $\mathrm{H}$ antigens and endotoxin. Blood 1996;88:3230-3239.

14 Bethge WA, Hegenbart U, Stuart MJ, Storer BE, Maris MB, Flowers ME, Maloney DG, Chauncey T, Bruno B, Agura E, Forman SJ, Blume KG, Niederwieser D, Storb R, Sandmaier BM: Adoptive immunotherapy with donor lymphocyte infusions after allogeneic hematopoietic cell transplantation following nonmyeloablative conditioning. Blood 2004;103:790-795.

15 Awwad M, North RJ: Cyclophosphamide (Cy)-facilitated adoptive immunotherapy of a Cy-resistant tumour. Evidence that Cy permits the expression of adoptive T-cell mediated immunity by removing suppressor $\mathrm{T}$ cells rather than by reducing tumour burden. Immunology 1988;65:87-92.

16 Bitan M, Or R, Shapira MY, Ackerstein A, Samuel S, Slavin S: Nonmyeloablative stem cell transplantation using lymphoablative rather than myeloablative conditioning in the prefludarabine era by ATG and limiting doses of cyclophosphamide. Bone Marrow Transplant 2005;35:953-958.

17 Phan GQ Rosenberg SA: Adoptive cell transfer for patients with metastatic melanoma: the potential and promise of cancer immunotherapy. Cancer Control 2013;20:289-297. 


\section{Cellular Physiology Cell Physiol Biochem 2015;35:2181-2191 \begin{tabular}{ll|l} 
and BOI: 10.1159/000374023 & $\begin{array}{l}\text { O 2015 S. Karger AG, Basel } \\
\text { www.karger.com/cpb }\end{array}$ \\
\hline
\end{tabular} \\ Zhu et al.: Antitumor Enhancement by Irradiated Haploidentical Donor Lymphocyte}

18 Strair RK, Schaar D, Medina D, Todd MB, Aisner J, DiPaola RS, Manago J, Knox B, Jenkinson A, Senzon R, Baker C, Liesel D, Ciardella M, Kuriyan M, Rubin A, Lattime EC: Antineoplastic effects of partially HLA-matched irradiated blood mononuclear cells in patients with renal cell carcinoma. J Clin Oncol 2003;21:3785-3791.

19 Bar M, Sandmaier BM, Inamoto Y, Bruno B, Hari P, Chauncey T, Martin PJ, Storb R, Maloney DG, Storer B, Flowers ME: Donor lymphocyte infusion for relapsed hematological malignancies after allogeneic hematopoietic cell transplantation: prognostic relevance of the initial CD3+ T cell dose. Biol Blood Marrow Transplan. 2013;19:949-957.

20 Kamiryo Y, Eto M, Yamada H, Yajima T, Harano M, Takeuchi A, Tatsugami K, Hamaguchi M, Naito S, Yoshikai Y: Donor CD4 T cells are critical in allogeneic stem cell transplantation against murine solid tumor. Cancer Res 2009;69:5151-5158.

21 Su X, Guo S, Zhou C, Wang D, Ma W, Zhang S: A simple and effective method for cancer immunotherapy by inactivated allogeneic leukocytes infusion. Int J Cancer 2009;124:1142-1151.

22 Morgan RA, Yang JC, Kitano M, Dudley ME, Laurencot CM, Rosenberg SA: Case report of a serious adverse event following the administration of $\mathrm{T}$ cells transduced with a chimeric antigen receptor recognizing ERBB2. Mol Ther 2010;18:843-851.

23 Lee DW, Gardner R, Porter DL, Louis CU, Ahmed N, Jensen M, Grupp SA, Mackall CL: Current concepts in the diagnosis and management of cytokine release syndrome. Blood 2014;124:188-195.

24 Willems L, Fevery S, Sprangers B, Rutgeerts O, Lenaerts C, Ibrahimi A, Gijsbers R, Van Gool S, Waer M, Billiau AD: Recipient leukocyte infusion enhances the local and systemic graft-versus-neuroblastoma effect of allogeneic bone marrow transplantation in mice. Cancer Immunol Immunother 2013;62:1733-1744.

25 Clark IA: The advent of the cytokine storm. Immunol Cell Biol 2007;85:271-273.

26 Boisgerault F, Anosova NG, Tam RC, Illigens BM, Fedoseyeva EV, Benichou G: Induction of T-cell response to cryptic MHC determinants during allograft rejection. Hum Immunol 2000;61:1352-1362.

27 Gould DS, Auchincloss H Jr: Direct and indirect recognition: the role of MHC antigens in graft rejection. Immunol Today 1999;20:77-82.

28 Roddie C, Peggs KS: Donor lymphocyte infusion following allogeneic hematopoietic stem cell transplantation. Expert Opin Biol Ther 2011;11:473-487. 